\title{
Continuous non-occlusive blood pressure monitoring at the sternum
}

\author{
M. Proença, A. Falhi, D. Ferrario, O. Grossenbacher, J.-A. Porchet, J. Krauss and J. Sola ${ }^{1}$ \\ ${ }^{1}$ Swiss Center for Electronics and Microtechnology (CSEM), Neuchâtel, Switzerland, josep.sola@csem.ch
}

\begin{abstract}
The clinical demand for a device to monitor Blood Pressure (BP) in ambulatory scenarios with minimal use of inflation cuffs is strong. Based on the so-called Pulse Wave Velocity (PWV) principle, a novel BP sensor that can be fully integrated in a shirt-like system has been developed. After a preliminary calibration, the sensor has shown to successfully estimate BP with an accuracy falling within the limits of the British Hypertensive Society (BHS) Grade A standard. The reported technique paves the way towards an ambulatory-compliant, continuous and non-invasive BP monitoring system, where the use of inflation cuffs is drastically reduced.
\end{abstract}

\section{Introduction}

Elevated Blood Pressure (BP) is a human-specific illness affecting a quarter of the worldwide population. Clinically known as hypertension, elevated BP is considered the major risk factor for cardiovascular disease: the most common cause of death in developed countries. Consequently, detecting, treating and controlling hypertension are major goals of modern medicine [8].

For more than one century, the non-invasive measurement of BP has relied on the inflation of pneumatic cuffs around a limb, typically the upper arm [9]. In addition to being occlusive, and thus cumbersome, clinical cuff-based methods provide intermittent $\mathrm{BP}$ readings, i.e. every twenty minutes, hence impeding the suitable monitoring of shortterm BP regulation mechanisms. In addition, cuff-based methods may not yield representative BP during sleep as repeated inflations induce arousal reactions, leading to non-representative overestimated BP values. Therefore, the development of novel technologies that reduce the recurrent use of pneumatic cuffs is clearly justified.

In this study a novel non-occlusive BP sensor that can be integrated in a shirt-like support is presented and validated.

\section{Methods}

This section introduces the working principles of the new BP sensor, specifies its current implantation, and reports on an in-vivo functional validation campaign.

\subsection{The Pulse Wave Velocity principle}

At each cardiac cycle, the opening of the aortic valve generates a pressure pulse that propagates along the walls of the entire arterial tree. The velocity at which this walldistending wave propagates is referred to as Pulse Wave Velocity (PWV), and in humans typically ranges from 4 $\mathrm{m} / \mathrm{s}$ in large elastic arteries, to $30 \mathrm{~m} / \mathrm{s}$ in small muscular arteries [1]. Already in 1905 it was observed that PWV depended on BP, and half a century later a mathematical model was proposed in order to describe such a relationship. So-called Moens-Korteweg equation, this model pre- dicts that PWV in an elastic tube (say, an artery) depends on the diameter $(d)$, thickness $(h)$ and stiffness $(E)$ of its wall, as well as on the density of the filling fluid $(\rho)$, i.e.:

$$
\mathrm{PWV}=\sqrt{\frac{h E}{d \rho}}
$$

In a living cardiovascular system, increasing BP will distend the walls of its elastic arteries, augmenting their stiffness, and increasing thus PWV. Since technological solutions to measure PWV in a continuous and non-occlusive manner already exist [2], PWV has become an appreciated surrogate measurement of BP [10]. Several implementations of such a system have been recently proposed: after a calibration procedure (i.e. a subject-dependent determination of $d, h$ and $\rho$ ) these techniques map PWV values measured at the ear lobes, the fingers or the phalanges [3] to the unknown underlying BP values.

Unfortunately, and as depicted by the Moens-Kortewerg model, the BP-PWV relationship is affected by several additional confounding factors, and a simple vasoconstrictor manoeuvre such as smoking a cigarette might modify it [4]. Because said confounding BP-PWV relationship is particularly accentuated in those segments of the arterial tree depicting vasomotion, attempts to measure PWV at distal peripheral arteries are irremediably associated to frequent re-calibration manoeuvres.

We suggested in [5] that assessing PWV in central elastic arteries would be a candidate solution to the exposed problematic. In particular, by measuring the propagation of pressure pulses along proximal segments of the aorta (i.e. along the ascending aorta), we expect to obtain pulse propagation properties of pure elastic arteries, minimizing then the need of vasomotion-induced recalibrations.

The current paper relates to a new technique to continuously assess PWV at central segments of the arterial tree by means of a comfortable and wearable monitoring system. Further post-processing of measured PWV values finally provides non-occlusive and reliable BP estimates. 


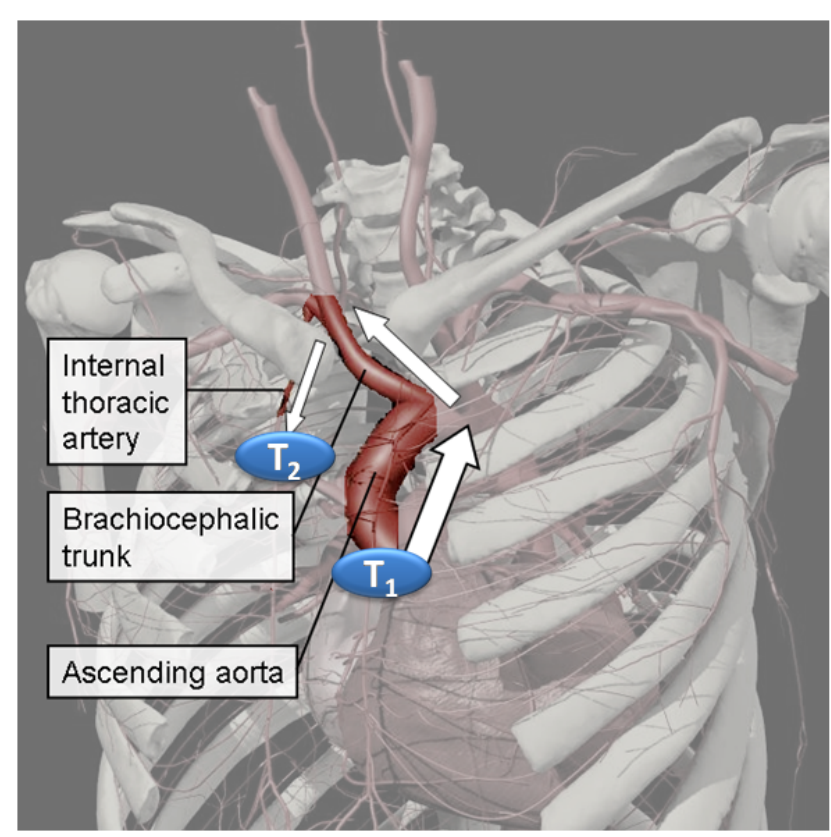

Figure 1 Arterial segments involved in the propagation of arterial pressure pulses for the chest sensor (from [6]).

\subsection{A blood pressure sensor at the chest}

In [6] we already described a wearable system for the monitoring of PWV in central arteries. In summary, the system estimates the Pulse Transit Time (PTT) of a pressure pulse travelling from the aortic valve towards the cutaneous vasculature on the sternum. Figure 1 illustrates the arterial segments involved in the propagation of such a pressure pulse. While the opening time of the aortic valve is determined by the joint analysis of an ElectroCardiogram (ECG), a Phono-Cardiogram (PCG) and a Impedance-Cardiogram (ICG), the arrival time at the sternum is measured by the processing of a multi-channel PhotoPlethysmogram (PPG). Figure 2 describes the approach.

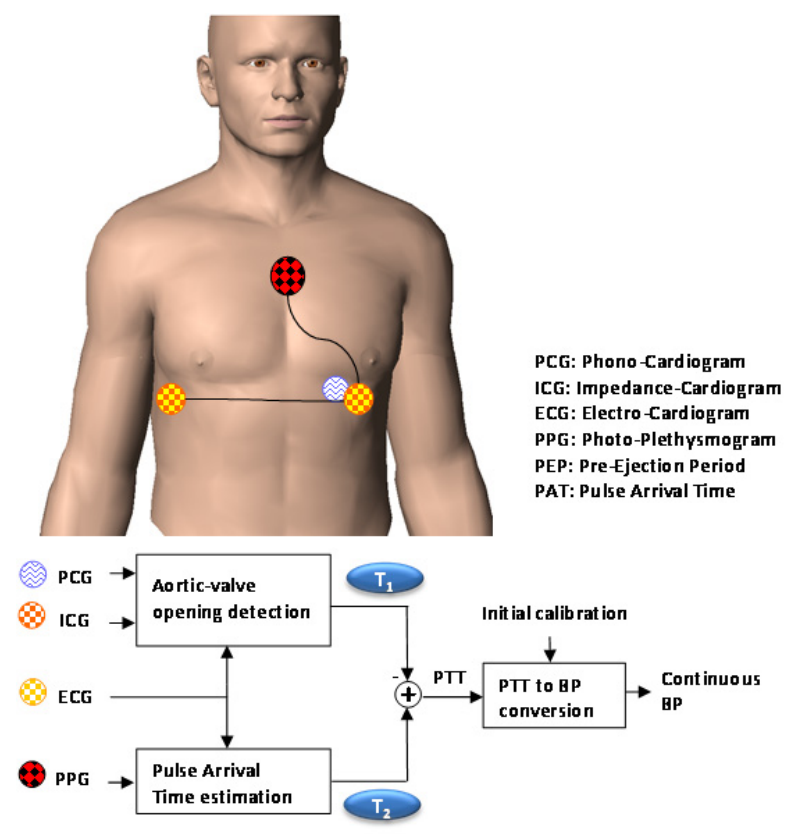

Figure 2 The novel wearable chest BP sensor.
The different sensing techniques involved in the wearable sensor are non-invasive and non-occlusive, thus continuously providing central PTT values without any cumbersomeness for the patient. In [6] it was also shown that PTT values provided by such a wearable technique highly correlate with reference aortic PTT measurements.

The current study goes one step forward in the path towards a continuous and non-occlusive BP sensor. In particular, this study tests the reliability of using continuous PTT measurements provided by the chest sensor to continuously estimate BP values. Initially a subject-dependent PTT-to-BP conversion function is estimated by means of a calibration manoeuvre involving the use of a reference occlusive BP device. Said function is then maintained and used to convert every measured PTT value into an estimated BP value (as in [7]). Note that in our case, since measured PTT values are associated to wall properties of elastic arteries only, said function is expected to be constant over time for each patient (i.e because of the minimized vasomotion activity).

\subsection{Validation}

A cohort of 8 young healthy subjects was asked to perform 3 left hand grip exercises spread over 25 minutes, at $40 \%$ of Maximum Voluntary Force (MVF). Reference Mean Arterial Pressure (MAP) values were recorded simultaneously with a Finapres system (Finapres; Ohmeda; Madison, WI) applied at the right-hand middle finger. Baseline MAP values for the entire cohort were of $82 \pm 13 \mathrm{mmHg}$, and hand grip exercises increased MAP to values up to 114 $\pm 8 \mathrm{mmHg}$. Simultaneously, every 18 seconds a single aortic PTT value was measured at the chest according to the method described in Section 2.2.

Because increased MAP is theoretically associated to decreased PTT values (see Section 2.1), both variables are expected to be negatively correlated. Accordingly, MAP and PTT time series were inspected via a Pearson correlation analysis.

Finally, in an offline procedure, six pairs of data points were selected within each subject's recording: each pair including a simultaneously-measured chest PTT value and its associated reference MAP value. Three pairs of data points corresponded to the lower measured MAP values, and three pairs to the higher measured MAP values. Said six pairs of data points were further used to estimate a subject-dependent PTT-to-BP conversion function for each subject, each conversion function being estimated as the $1^{\text {st }}$ order polynomial function $f$ that satisfied:

$$
\operatorname{argmin}_{f} \sum_{i}\left[\mathrm{MAP}_{i}-f\left(\mathrm{PTT}_{i}\right)\right]^{2}
$$

Each subject-dependent conversion function was finally applied to its associated series of PTT values, providing a new series of continuous BP estimates. Figures of merit of the reliability of the novel wearable chest sensor to continuously estimate BP along the entire experiment were finally obtained by comparing said estimated BP values to their simultaneously-measured finger MAP values. 

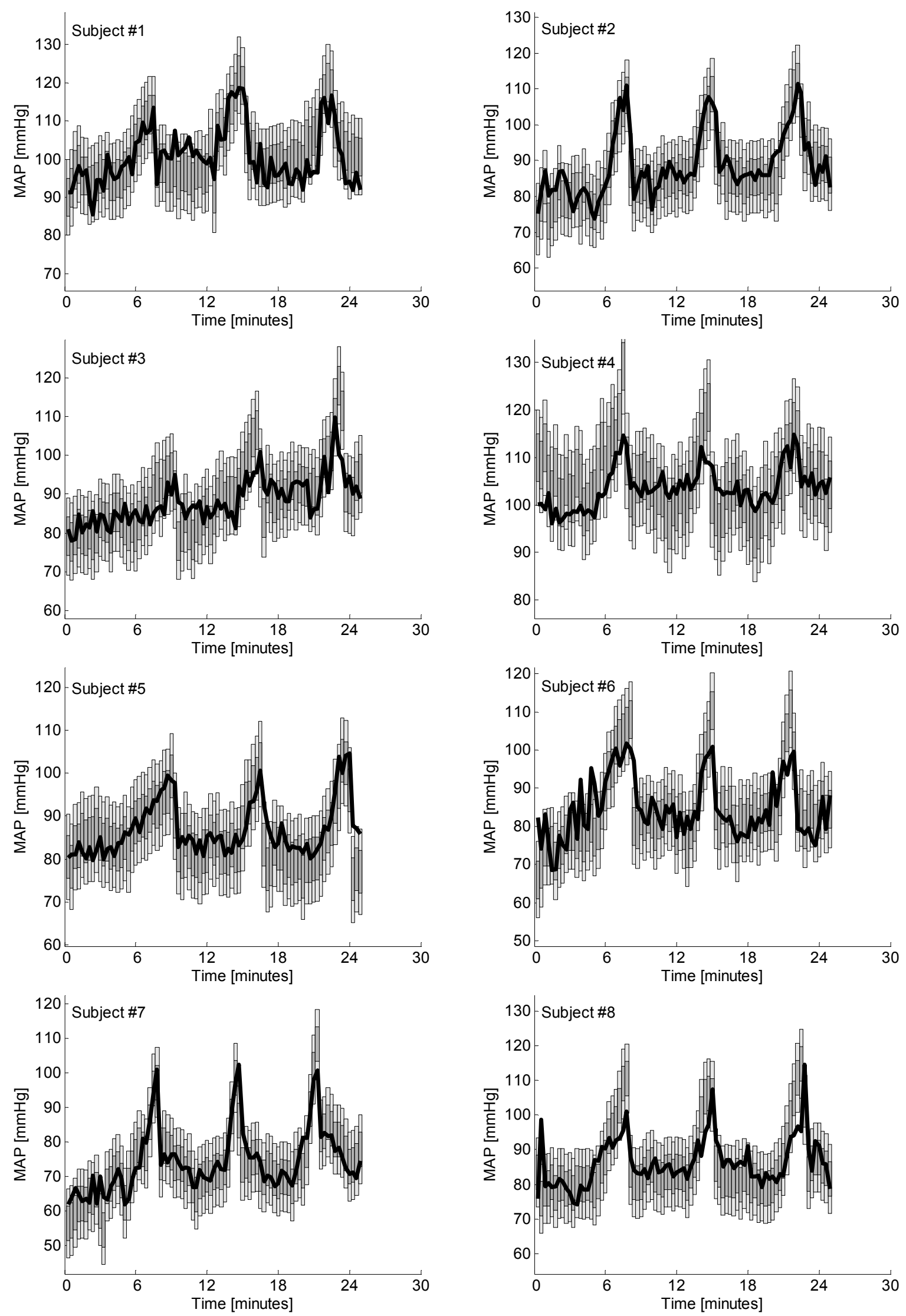

Figure 3 Results for the 8 subjects involved in the validation campaign. Bold lines depict Mean Arterial Pressure (MAP) values as estimated by the non-occlusive chest sensor. Dark and white boxes depict respectively $\pm 5 \mathrm{mmHg}$ and $\pm 10 \mathrm{mmHg}$ confidence intervals as provided by the reference occlusive BP device. 


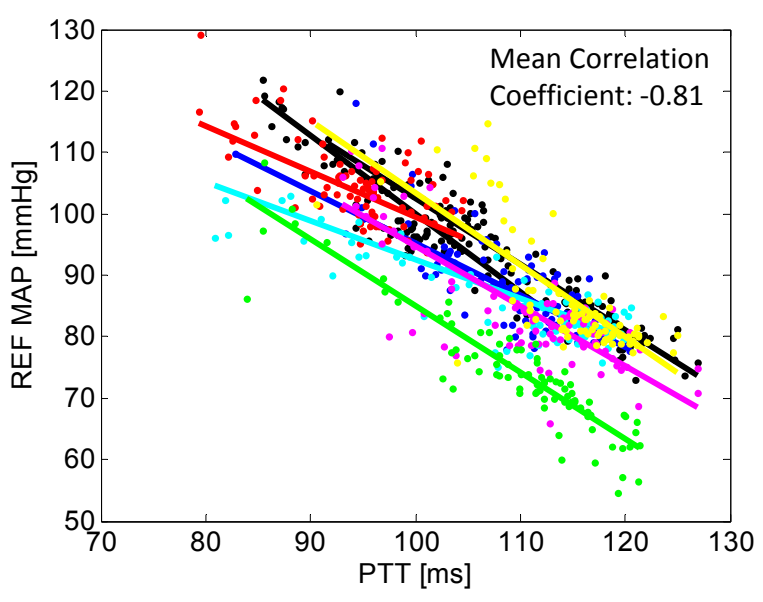

Figure 4 Correlation plot between chest PTT and reference MAP. Different colors depict different subjects.

\section{Results}

According to the validation procedure established in Section 2.3, and by using the experimental setup described in Section 2.2, the feasibility of continuously measuring BP by means of a wearable non-occlusive chest sensor is here reported.

Concerning the Pearson correlation analysis, an average correlation coefficient of $r=-0.81$ (range of $[-0.64 ;-0.92]$ ) between chest PTT measurements and reference MAP values was obtained. Figure 4 illustrates correlation plots for each of the subjects in the cohort.

Concerning the estimation of PTT-based BP values according to subject-dependent conversion functions, the percentage of estimated MAP values falling within a $\pm 5 \mathrm{mmHg}$ confidence interval compared to the reference measurements was of $76.1 \%$ (range of $[67.5 ; 85.5] \%$ ). The percentage of values falling within a confidence interval of $\pm 10 \mathrm{mmHg}$ was of $94.9 \%$ (range of $[89.2 ; 100.0] \%$ ). Figure 3 illustrates MAP estimation results for each subject in the cohort.

Note that according to the British Hypertension Society, a BP monitoring device is considered of Grade A if the percentages of values falling within $\pm 5 \mathrm{mmHg}$ and $\pm 10 \mathrm{mmHg}$ confidence intervals are greater than $60 \%$ and $85 \%$ respectively.

\section{Conclusion}

The issue of monitoring Blood Pressure (BP) in a continuous and non-occlusive way has been addressed via a chest sensor that is able to measure the pressure Pulse Transit Times (PTT) from the aortic valve to the sternum vascular bed. In a cohort of 8 subjects, said PTT values have been shown to strongly correlate with simultaneously-recorded reference Mean Arterial Pressure (MAP) values. Additionally, the chest BP sensor has proved to provide MAP values complying with the BHS Grade A standard.

These promising results however need to be further investigated and confirmed in larger clinical studies, involving broader population cohorts and additional hypertensive models. The actual implementation of a subject-dependent calibration function requires additional research too.

All in all, the reported technique paves the way towards a fully ambulatory compliant, continuous and non-invasive BP monitoring system, where the use of inflation cuffs is minimal.

\section{$4 \quad$ References}

[1] W. W. Nichols and M. F. O'Rourke, McDonald's Blood Flow in Arteries. Oxford: Oxford University Press, 2005.

[2] J. Solà, et al., "Ambulatory monitoring of the cardiovascular system: the role of Pulse Wave Velocity," in New Developments in Biomedical Engineering, D. Campolo, Ed., ed Vienna:: I-Tech Education and Publishing, 2010

[3] J. Solà, "Continuous non-invasive blood pressure estimation", ETHZ PhD Dissertation No. 20093, Dr. Sc., 30 November 2011

[4] J. Solà, et al., "Continuous monitoring of cardiovascular responses", Proc. EMBS 2008, 1423-1426, Vancouver, IEEE, 2008.

[5] J. Solà, et al., "Non-invasive monitoring of central blood pressure by Electrical Impedance Tomography (EIT): first experimental evidence", Med Biol Eng Comput, Vol 49, Num 4, 409-415, 201.

[6] J. Solà, et al., "Chest Pulse Wave Velocity: a Novel Approach to Assess Arterial Stiffness", IEEE Transactions on Biomedical Engineering, 58, 215-223, 2010.

[7] W. Chen, et al., "Continuous estimation of systolic blood pressure using the pulse arrival time and intermittent calibration," Med Biol Eng Comput, vol. 38, pp. 569-74, 2000.

[8] G. Mancia, et al., "2007 Guidelines for the management of arterial hypertension: The Task Force for the Management of Arterial Hypertension of the European Society of Hypertension (ESH) and of the European Society of Cardiology (ESC)," Eur Heart J, vol. 28, pp. 1462-536, Jun 2007.

[9] L. A. Geddes, Handbook of Blood Pressure Measurement: Humana Press, 1991.

[10] G. Zhang, et al., "Pulse arrival time is not an adequate surrogate for pulse transit time as a marker of blood pressure", Journal of Applied Physiology December 1, vol. 111 no. 6 1681-1686, 2011. 